\title{
Identifying the Optical Response of Graphene Using Electron Energy-Loss Spectroscopy
}

Juan-Carlos Idrobo ${ }^{1}$, Florence J. Nelson ${ }^{2}$, Zoran L. Mišković ${ }^{3}$, Alain C. Diebold ${ }^{3}$, Stephen J. Pennycook $^{1,2}$, and Sokrates T. Pantelides ${ }^{4,1}$

${ }^{1}$ Materials Science \& Technology Division, Oak Ridge National Laboratory, Oak Ridge, TN 37831, USA

${ }^{2}$ College of Nanoscale Science and Engineering, University at Albany, 255 Fuller Rd., Albany, New York 12203, USA.

${ }^{3}$ Department of Applied Mathematics, University of Waterloo, Waterloo, Ontario, Canada N2L 3G1.

${ }^{4}$ Dept. of Physics \& Astronomy, Vanderbilt University, Nashville, TN 37235, USA

Graphene, due to its intrinsic two-dimensional and flexible structure, excellent electronic transport and unusual optical properties is believed to be an ideal candidate material for the next generation of plasmonic devices. Yet, despite previous studies of the energy loss function of monolayer graphene [1], there has not been a study that actually shows that the optical response of clean, freely suspended graphene has singularities and thus presents plasmons.

In this talk, we will present the dielectric function of monolayer graphene obtained by electron energy-loss spectroscopy (EELS) in a low-voltage aberration-corrected scanning transmission electron microscope (STEM). We will discuss in detail the methods to obtain the optical response of two-dimensional materials from EELS experiments, and present further insights into whether the optical response of graphene is formed by plasmons or other electronic excitations [4].

\section{References}

[1] M H Gass, et al., Nat. Nanotech. 3, (2008) p. 676; T Eberlein, et al. Phys. Rev. B 77, (2008) p. 233406; W Zhou et al., Nat. Nanotech. 7, (2012) p. 161.

[2] This work was funded by the Institute for Nanoelectronics Discovery and Exploration (INDEX)/Nanoelectronics Research Initiative (NRI) and the New York Center for National Competitiveness in Nanoscale Characterization (NC3), by Oak Ridge National Laboratory's Shared Research Equipment (ShaRE) User Program (JCI), which is sponsored by the Office of Basic Energy Sciences, U.S. Department of Energy; the Materials Sciences and Engineering Division, Office of Basic Energy Sciences, U.S. Department of Energy (SJP, STP). 


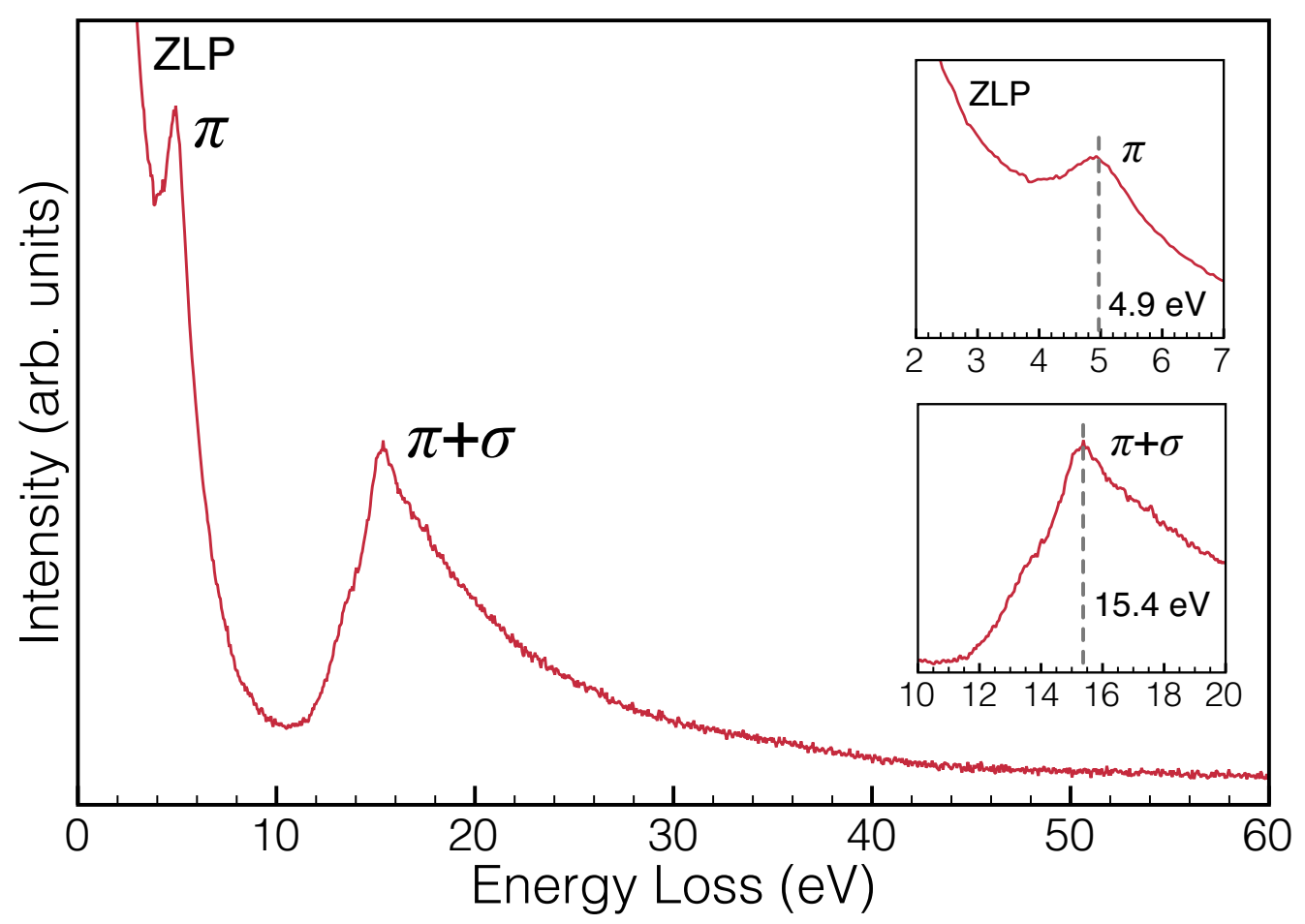

FIG. 1. EELS spectrum of monolayer graphene measured up to $60 \mathrm{eV}$. Three main features are visible in the spectrum, the zero loss peak (ZLP), which is composed of electron that do not go through inelastic scattering, and two absorption peaks labeled $\pi$ and $\pi+\sigma$. The insets highlight the position and shape of the $\pi$ and $\pi+\sigma$ peaks. The spectra were acquired while scanning the atomic-size electron beam in a clean region, free of defects, with an area of $8 \times 8$ $\mathrm{nm}^{2}$. 